Technical Report No. 6/04, September 2004 CONCOMITANTS OF GENERALIZED ORDER STATISTICS FROM FARLIE-GUMBEL-MORGENSTERN DISTRIBUTIONS

M.I. Beg and M. Ahsanullah 


\title{
CONCOMITANTS OF GENERALIZED ORDER STATISTICS FROM FARLIE-GUMBEL-MORGENSTERN DISTRIBUTIONS
}

\author{
M.I. BEG ${ }^{1}$ AND M. AHSANULLAH \\ University of Hyderabad and Rider University
}

Generalized order statistics constitute a unified model for ordered random variables that includes order statistics and record values among others. Here, we consider concomitants of generalized order statistics for the Farlie-GumbelMorgenstern bivariate distributions and study recurrence relations between their moments. We derive the joint distribution of concomitants of two generalized order statistics and obtain their product moments. Application of these results is seen in establishing some well known results given separately for order statistics and record values and obtaining some new results.

1. Introduction. The Farlie-Gumbel-Morgenstern (FGM) family of bivariate distributions has found extensive use in practice. This family is characterized by the specified marginal distribution functions $F_{X}(x)$ and $F_{Y}(y)$ of random variables $X$ and $Y$, respectively, and a parameter $\alpha$, resulting in the bivariate distribution function (df) given by

$$
F_{X, Y}(x, y)=F_{X}(x) F_{Y}(y)\left[1+\alpha\left\{1-F_{X}(x)\right\}\left\{1-F_{Y}(y)\right\}\right]
$$

with the corresponding probability density function (pdf)

$$
f_{X, Y}(x, y)=f_{X}(x) f_{Y}(y)\left[1+\alpha\left\{2 F_{X}(x)-1\right\}\left\{2 F_{Y}(y)-1\right\}\right] .
$$

Here $f_{X}(x)$ and $f_{Y}(y)$ are the marginals of $f_{X, Y}(x, y)$. The parameter $\alpha$ is known as the association paprameter, the two random variables $X$ and $Y$ are independent when $\alpha$ is zero. Such a model was originally introduced by Morgenstern (1956) and investigated by Gumbel (1960) for exponential marginals, the general form in (1.1) is due to Farlie (1960) and Johnson and Kotz (1975). The admissible range of association paprameter $\alpha$ is $-1 \leq \alpha \leq$ 1 and the Pearson correlation coefficient $\rho$ between $X$ and $Y$ can never

${ }^{1}$ Currently on sabbatical at Concordia University, Canada. AMS 2000 subject classification. 62G30.

Key words and phrases. Generalized order statistics, order statistics, record values, concomitants, recurrence relations. 
exceed $1 / 3$. The conditional distribution function (df) and probability density function (pdf) of $Y$, given $X$, are respectively,

$$
F_{Y \mid X}(y \mid x)=F_{Y}(y)\left[1+\alpha\left\{1-2 F_{X}(x)\right\}\left\{1-F_{Y}(y)\right\}\right]
$$

and

$$
f_{Y \mid X}(x \mid y)=f_{Y}(y)\left[1+\alpha\left\{2 F_{X}(x)-1\right\}\left\{2 F_{Y}(y)-1\right\}\right] .
$$

Suppose $X(1, n, m, k), X(2, n, m, k), \ldots, X(n, n, m, k)(k \geq 1, m$ is a real number $\geq-1)$, are $n$ generalized order statistics from an absolutely continuous (with respect to Lebesgue measure) df $F(x)$ and pdf $f(x)$. Their joint pdf $f_{1,2, \ldots, n}\left(x_{1}, x_{2}, \ldots, x_{n}\right)$ can be written as [see Kamps (1995), pp. 50-51]

$$
f_{1,2, \ldots, n}\left(x_{1}, x_{2}, \ldots, x_{n}\right)=\left\{\begin{array}{l}
k \prod_{j=1}^{n-1} \gamma_{j} \prod_{i=1}^{n-1}\left(\bar{F}\left(x_{i}\right)\right)^{m} f\left(x_{i}\right)\left(\bar{F}\left(x_{n}\right)\right)^{k-1} f\left(x_{n}\right), \\
F^{-1}(0)<x_{1}<x_{2}<\ldots<x_{n}<F^{-1}(1) \\
0, \text { otherwise }
\end{array}\right.
$$

where $\bar{F}(x)=1-F(x)$ and $\gamma_{j}=k+(n-j)(m+1), j=1,2, \cdots, n$.

The generalized order statistics were introduced by Kamps (1995) as a unified model for ordered random variables which includes among others order statistics, record values and $k$-record values as special cases. If $m=0$ and $k=1$, then $X(r, n, m, k)$ reduces to the $r$-th order statistic and (1.5) gives the joint pdf of the $n$ order statistics $X_{1, n} \leq X_{2, n} \leq \ldots \leq X_{n, n}$. If $k=1$ and $m=-1$, then (1.5) gives the joint pdf of the first $n$ upper record values from a sequence of iid random variables with df $F(x)$ and pdf $f(x)$. For details of order statistics and upper record values, the reader may refer to David and Nagaraja (2003) and Ahsanullah (2004), respectively.

Integrating out $x_{1}, x_{2}, \ldots, x_{r-1}, x_{r+1}, \ldots$, and $x_{n}$ from (1.5), we get the pdf $f_{r, n, m, k}(x)$ of $X(r, n, m, k), 1 \leq r \leq n$ [see Kamps (1995), p. 64] as

$$
f_{r, n, m, k}(x)=\frac{c_{r-1}}{(r-1) !}(\bar{F}(x))^{\gamma_{r}-1} f(x) g_{m}^{r-1}(F(x)),
$$

where $c_{r-1}=\prod_{j=1}^{r} \gamma_{j}$,

$$
g_{m}(x)=h_{m}(x)-h_{m}(0)= \begin{cases}\frac{1}{m+1}\left(1-(1-x)^{m+1}\right), & m \neq-1 \\ -\ln (1-x), & m=-1, x \in[0,1)\end{cases}
$$


and

$$
h_{m}(x)= \begin{cases}-\frac{1}{m+1}(1-x)^{m+1}, & m \neq-1 \\ -\ln (1-x), & m=-1, x \in[0,1) .\end{cases}
$$

Note, since $\lim _{m \rightarrow-1}\left[\frac{1}{(m+1)}\left(1-(1-x)^{m+1}\right)\right]=-\ln (1-x)$, we will write $g_{m}(x)=\left[\frac{1}{(m+1)}\left(1-(1-x)^{m+1}\right)\right]$, for all $x \in[0,1)$ and for all $m$ with $g_{-1}(x)=$ $\lim _{m \rightarrow-1} g_{m}(x)$.

The joint pdf of $X(r, n, m, k)$ and $X(s, n, m, k), 1 \leq r<s \leq n$, is given by [see Kamps (1995), p. 68]

$$
\begin{aligned}
f_{r, s, n, m, k}(x, y)= & \frac{c_{s-1}}{(r-1) !(s-r-1) !}(\bar{F}(x))^{m} f(x) g_{m}^{r-1}(F(x)) \\
& \times\left[h_{m}(F(y))-h_{m}(F(x))\right]^{s-r-1}(\bar{F}(y))^{\gamma_{s}-1} f(y), x<y .
\end{aligned}
$$

For more details of generalized order statistics the reader is reffered to the monograph of Kamps (1995).

Let $\left(X_{i}, Y_{i}\right), i=1,2, \ldots$ be a sequence of $i i d$ bivariate random variables $(X, Y)$ with an absolutely continuous (with respect to Lebesgue measure) df $F_{X, Y}(x, y)$. Denote by $Y_{[r, n, m, k]}, 1 \leq r \leq n$ the $Y$ value associated with $X(r, n, m, k)$. We call $Y_{[r, n, m, k]}$ the concomitant of the $r$-th generalized order statistic. The pdf and df of $Y_{[r, n, m, k]}, 1 \leq r \leq n$, denoted by $g_{[r, n, m, k]}(y)$ and $G_{[r, n, m, k]}(y)$, respectively are given by

$$
g_{[r, n, m, k]}(y)=\int_{-\infty}^{\infty} f_{Y \mid X}(y \mid x) f_{r, n, m, k}(x) d x
$$

and

$$
G_{[r, n, m, k]}(y)=\int_{-\infty}^{\infty} F_{Y \mid X}(y \mid x) f_{r, n, m, k}(x) d x,
$$

where $f_{r, n, m, k}(x)$ is the pdf of $X(r, n, m, k), 1 \leq r \leq n$. An excellent review on concomitant of order statistics is given in David and Nagaraja (1998) and Bhattacharya (1984).

In this paper, we study the properties of $Y_{[r, n, m, k]}$ associated with the FGM distributions given by (1.1) and obtain recurrence relations between moments and moment generating functions (mgf) of concomitants. Finally, we present the joint distribution of concomitants of two generalized order statistics and their product moments.

2. Concomitants of generalized order statistics. For the FGM distributions with pdf given by (1.2), utilizing (1.4) and (1.6) in (1.8), one 
obtains the pdf of $Y_{[r, n, m, k]}$

(2.1)

$$
\begin{aligned}
g_{[r, n, m, k]}(y)= & \int_{-\infty}^{\infty} f_{Y}(y)\left[1-\alpha\left\{1-2 F_{X}(x)\right\}\left\{2 F_{Y}(y)-1\right\}\right] \\
& \times \frac{c_{r-1}}{(r-1) !}\left(\bar{F}_{X}(x)\right)^{\gamma_{r}-1}\left[\frac{1}{m+1}\left(1-(\bar{F}(x))^{m+1}\right)\right]^{r-1} f_{X}(x) d x \\
= & f_{Y}(y)-\alpha\left(2 F_{Y}(y)-1\right) f_{Y}(y)\left[1-\frac{2 c_{r-1}}{(r-1) !} \int_{-\infty}^{\infty} F_{X}(x)(\bar{F}(x))^{\gamma_{r}-1}\right. \\
& \left.\times\left\{\frac{1}{m+1}\left(1-(\bar{F}(x))^{m+1}\right)\right\}^{r-1} f_{X}(x) d x\right] .
\end{aligned}
$$

Consider

$$
\begin{aligned}
I & =\frac{c_{r-1}}{(r-1) !} \int_{-\infty}^{\infty} F_{X}(x)\left(\bar{F}_{X}(x)\right)^{\gamma_{r}-1}\left[\frac{1}{m+1}\left(1-\left(\bar{F}_{X}(x)\right)^{m+1}\right)\right]^{r-1} f_{X}(x) d x \\
& =1-\frac{c_{r-1}}{(r-1) !} \int_{-\infty}^{\infty}\left(\bar{F}_{X}(x)\right)^{\gamma_{r}}\left[\frac{1}{m+1}\left(1-\left(\bar{F}_{X}(x)\right)^{m+1}\right)\right]^{r-1} f_{X}(x) d x .
\end{aligned}
$$

Making transformation $u=\bar{F}_{X}(x)$, we get

$$
I=1-\frac{c_{r-1}}{(r-1) !} \int_{0}^{1} u^{\gamma_{r}}\left[\frac{1}{m+1}\left(1-u^{m+1}\right)\right]^{r-1} d u .
$$

Further, making transformation $t=1-u^{m+1}$, we get

$$
\begin{aligned}
& I=1-\frac{c_{r-1}}{(r-1) !(m+1)^{r}} \int_{0}^{1} t^{r-1}(1-t)^{\frac{\gamma_{r}-m}{m+1}} d t \\
& =1-\frac{c_{r-1}}{(r-1) !(m+1)^{r}} B\left(r, \frac{\gamma_{r}-m}{m+1}+1\right) \\
& =1-\frac{c_{r-1}}{(r-1) !(m+1)^{r}} B\left(r, \frac{\gamma_{r-1}-m}{m+1}\right), \text { using, } \gamma_{r}=\gamma_{r-1}-(m+1) \\
& =1-\frac{c_{r-1}}{(r-1) !(m+1)^{r}} \frac{\Gamma(r) \Gamma\left(\frac{\gamma_{r-1}-m}{m+1}\right)}{\Gamma\left(r+\frac{\gamma_{r-1}-m}{m+1}\right)} \\
& =1-\frac{c_{r-1}}{(r-1) !(m+1)^{r}} \frac{(r-1) ! \Gamma\left(\frac{\gamma_{r-1}-m}{m+1}\right)}{\left(r+\frac{\gamma_{r-1}-m}{m+1}-1\right)\left(r+\frac{\gamma_{r-1}-m}{m+1}-2\right) \cdots\left(\frac{\gamma_{r-1}-m}{m+1}\right) \Gamma\left(\frac{\gamma_{r-1}-m}{m+1}\right)} \\
& =1-\frac{c_{r-1}}{\left(\gamma_{1}+1\right)\left(\gamma_{2}+1\right) \cdots\left(\gamma_{r}+1\right)} \\
& =1-C(r, n, m, k), \text { say }
\end{aligned}
$$

Hence, from (2.1), we get

$$
\begin{aligned}
g_{[r, n, m, k]}(y) & =f_{Y}(y)-\alpha\left(2 F_{Y}(y)-1\right) f_{Y}(y)[1-2(1-C(r, n, m, k))] \\
& =f_{Y}(y)+\alpha\left(2 F_{Y}(y)-1\right) f_{Y}(y) C^{*}(r, n, m, k),
\end{aligned}
$$


where $C^{*}(r, n, m, k)=1-2 C(r, n, m, k)$. The above expression of $g_{[r, n, m, k]}(y)$ does not depend on $F_{X}(x)$ at all! Observing that $2 F_{Y}(y) f_{Y}(y)$ is the pdf of $Y_{2,2}$, the second order statistic of a random sample of size two of $Y$ variate, We find that the distribution of the $r$-th concomitant depends only on the marginal distribution of $Y$ and the distribution of $Y_{2,2}$. From (2.2) we now have

$$
g_{[r, n, m, k]}(y)=f_{Y_{1,1}}(y)+\alpha C^{*}(r, n, m, k)\left[f_{Y_{2,2}}(y)-f_{Y_{1,1}}(y)\right] .
$$

Writing $2 F_{Y}(y)-1=F_{Y}(y)+F_{Y}(y)-1$, in $(2.2)$, we obtain

$$
g_{[r, n, m, k]}(y)=f_{Y_{1,1}}(y)-\frac{\alpha}{2} C^{*}(r, n, m, k)\left[f_{Y_{1,2}}(y)-f_{Y_{2,2}}(y)\right] .
$$

3. Moments and moment generating function of concomitants. Using the results of the previous section, we derive the moments and mgf of $Y_{[r, n, m, k]}$ as follows.

From (2.3), the $l$-th moment of $Y_{[r, n, m, k]}$ is

$$
\begin{aligned}
\mu_{[r, n, m, k]}^{(l)} & =E\left\{Y_{[r, n, m, k]}^{l}\right\}=\int_{-\infty}^{\infty} y^{l} g_{[r, n, m, k]}(y) d y \\
& =\left(1-\alpha C^{*}(r, n, m, k)\right) \mu_{1,1}^{(l)}+\alpha C^{*}(r, n, m, k) \mu_{2,2}^{(l)},
\end{aligned}
$$

where $\mu_{1,1}^{(l)}=E\left\{Y^{l}\right\}$ and $\mu_{2,2}^{(l)}=E\left\{Y_{2,2}^{l}\right\}$. Thus $\mu_{[r, n, m, k]}^{(l)}$ is known for all $r, n, m$ and $k$ if we know $\mu_{1,1}^{(l)}$ and $\mu_{2,2}^{(l)}$. In general, if $h(y)$ is a measurable function of $y$, then

$E\left\{h\left(Y_{[r, n, m, k]}\right\}=\left(1-\alpha C^{*}(r, n, m, k)\right) E\left\{h\left(Y_{1,1}\right)\right\}+\alpha C^{*}(r, n, m, k) E\left\{h\left(Y_{2,2}\right)\right\}\right.$,

provided the expectations exist.

In particular, the mgf of $Y_{[r, n, m, k]}$ is given by

$$
M_{[r, n, m, k]}(t)=\left(1-\alpha C^{*}(r, n, m, k)\right) M_{1,1}(t)+\alpha C^{*}(r, n, m, k) M_{2,2}(t),
$$

where $M_{1,1}(t)=E\{\exp (t Y)\}$ and $M_{2,2}(t)=E\left\{\exp \left(t Y_{2,2}\right)\right\}$.

4. Recurrence relation between moments of concomitants. In this section we shall present several recurrence relations between pdf's, moments and mgf's of concomitants. From (2.34), we have

$$
g_{[r, n-1, m, k]}(y)=f_{Y_{1,1}}(y)+\alpha C^{*}(r, n-1, m, k)\left[f_{Y_{2,2}}(y)-f_{Y_{1,1}}(y)\right] .
$$


Evidently

(4.2)

$$
\begin{aligned}
g_{[r, n, m, k]}(y)-g_{[r, n-1, m, k]}(y)= & \alpha\left[C^{*}(r, n, m, k)-C^{*}(r, n-1, m, k)\right] \\
& \times\left[f_{Y_{2,2}}(y)-f_{Y_{1,1}}(y)\right] .
\end{aligned}
$$

It follows from (2.4) that

$$
g_{[r-1, n, m, k]}(y)=f_{Y_{1,1}}(y)+\alpha C^{*}(r-1, n, m, k)\left[f_{Y_{2,2}}(y)-f_{Y_{1,1}}(y)\right] .
$$

Consider the difference

$$
\begin{aligned}
g_{[r, n, m, k]}(y)-g_{[r-1, n, m, k]}(y)= & \alpha\left[C^{*}(r, n, m, k)-C^{*}(r-1, n, m, k)\right] \\
& \times\left[f_{Y_{2,2}}(y)-f_{Y_{1,1}}(y)\right] .
\end{aligned}
$$

Analogously, one can write for $1 \leq i_{1} \leq n-r$

$$
\begin{aligned}
g_{[r, n, m, k]}(y)-g_{\left[r, n-i_{1}, m, k\right]}(y)= & \alpha\left[C^{*}(r, n, m, k)-C^{*}\left(r, n-i_{1}, m, k\right)\right] \\
& \times\left[f_{Y_{2,2}}(y)-f_{Y_{1,1}}(y)\right]
\end{aligned}
$$

and for $1 \leq j_{1} \leq r-1$

(4.6)

$$
\begin{aligned}
g_{[r, n, m, k]}(y)-g_{\left[r-j_{1}, n, m, k\right]}(y)= & \alpha\left[C^{*}(r, n, m, k)-C^{*}\left(r-j_{1}, n, m, k\right)\right] \\
& \times\left[f_{Y_{2,2}}(y)-f_{Y_{1,1}}(y)\right] .
\end{aligned}
$$

For $1 \leq i_{1} \leq i_{2} \leq n-r$ and $1 \leq j_{1} \leq j_{2} \leq r-1$ one has

$$
\begin{aligned}
g_{[r, n, m, k]}(y)-g_{\left[r-j_{1}, n-i_{1}, m, k\right]}(y)= & \alpha\left[C^{*}(r, n, m, k)-C^{*}\left(r-j_{1}, n-i_{1}, m, k\right)\right] \\
& \times\left[f_{Y_{2,2}}(y)-f_{Y_{1,1}}(y)\right]
\end{aligned}
$$

and

$$
\begin{aligned}
g_{\left[r-j_{1}, n-i_{1}, m, k\right]}(y) & -g_{\left[r-j_{2}, n-i_{2}, m, k\right]}(y)=\alpha\left[C^{*}\left(r-j_{1}, n-i_{1}, m, k\right)\right. \\
& \left.-C^{*}\left(r-j_{2}, n-i_{2}, m, k\right)\right]\left[f_{Y_{2,2}}(y)-f_{Y_{1,1}}(y)\right] .
\end{aligned}
$$

Utilizing equations (4.5)-(4.8), we have the following theorems.

THEOREM 4.1. Let $1 \leq i_{1} \leq i_{2} \leq n-r$ and $1 \leq j_{1} \leq j_{2} \leq r-1$. For $a$ bivariate random variable $(X, Y)$ having pdf (1.2), the following recurrence relations between moments of concomitants are valid:

$$
\begin{aligned}
\mu_{[r, n, m, k]}^{(l)}-\mu_{\left[r, n-i_{1}, m, k\right]}^{(l)}= & \alpha\left[C^{*}(r, n, m, k)-C^{*}\left(r, n-i_{1}, m, k\right)\right] \\
& \times\left[\mu_{2,2}^{(l)}-\mu_{1,1}^{(l)}\right]
\end{aligned}
$$




$$
\begin{aligned}
\mu_{[r, n, m, k]}^{(l)}-\mu_{\left[r-j_{1}, n, m, k\right]}^{(l)}= & \alpha\left[C^{*}(r, n, m, k)-C^{*}\left(r-j_{1}, n, m, k\right)\right] \\
& \times\left[\mu_{2,2}^{(l)}-\mu_{1,1}^{(l)}\right]
\end{aligned}
$$

and

$(4.11)$

$$
\begin{aligned}
\mu_{[r, n, m, k]}^{(l)}-\mu_{\left[r-j_{1}, n-i_{1}, m, k\right]}^{(l)}= & \alpha\left[C^{*}(r, n, m, k)-C^{*}\left(r-j_{1}, n-i_{1}, m, k\right)\right] \\
& \times\left[\mu_{2,2}^{(l)}-\mu_{1,1}^{(l)}\right] .
\end{aligned}
$$

In general it is true that

$$
\begin{aligned}
\mu_{\left[r-j_{1}, n-i_{1}, m, k\right]}^{(l)} & -\mu_{\left[r-j_{2}, n-i_{2}, m, k\right]}^{(l)}=\alpha\left[C^{*}\left(r-j_{1}, n-i_{1}, m, k\right)\right. \\
& \left.-C^{*}\left(r-j_{2}, n-i_{2}, m, k\right)\right]\left[\mu_{2,2}^{(l)}-\mu_{1,1}^{(l)}\right] .
\end{aligned}
$$

THEOREM 4.2. Under the conditions of Theorem 4.1, the relation between mgf's of concomitants are

$$
\begin{aligned}
M_{[r, n, m, k]}(t)-M_{\left[r, n-i_{1}, m, k\right]}(t)= & \alpha\left[C^{*}(r, n, m, k)-C^{*}\left(r, n-i_{1}, m, k\right)\right] \\
& \times\left[M_{2,2}(t)-M_{1,1}(t)\right]
\end{aligned}
$$

$$
\begin{aligned}
M_{[r, n, m, k]}(t)-M_{\left[r-j_{1}, n, m, k\right]}(t)= & \alpha\left[C^{*}(r, n, m, k)-C^{*}\left(r-j_{1}, n, m, k\right)\right] \\
& \times\left[M_{2,2}(t)-M_{1,1}(t)\right]
\end{aligned}
$$

and

(4.15)

$$
\begin{aligned}
M_{[r, n, m, k]}(t)-M_{\left[r-j_{1}, n-i_{1}, m, k\right]}(t)= & \alpha\left[C^{*}(r, n, m, k)-C^{*}\left(r-j_{1}, n-i_{1}, m, k\right)\right] \\
& \times\left[M_{2,2}(t)-M_{1,1}(t)\right] .
\end{aligned}
$$

In general

(4.16)

$$
\begin{aligned}
M_{\left[r-j_{1}, n-i_{1}, m, k\right]}(t) & -M_{\left[r-j_{2}, n-i_{2}, m, k\right]}(t)=\alpha\left[C^{*}\left(r-j_{1}, n-i_{1}, m, k\right)\right. \\
& \left.-C^{*}\left(r-j_{2}, n-i_{2}, m, k\right)\right]\left[M_{2,2}(t)-M_{1,1}(t)\right] .
\end{aligned}
$$

We have from (4.4),

$$
\begin{aligned}
g_{[r, n, m, k]}(y)- & g_{[r-1, n, m, k]}(y)=\alpha\left[C^{*}(r, n, m, k)-C^{*}(r-1, n, m, k)\right] \\
& \times\left[f_{Y_{2,2}}(y)-f_{Y_{1,1}}(y)\right]
\end{aligned}
$$




$$
\begin{aligned}
& =\alpha\left[\left\{1-2 \frac{\gamma_{1} \gamma_{2} \cdots \gamma_{r}}{\left(\gamma_{1}+1\right)\left(\gamma_{2}+1\right) \cdots\left(\gamma_{r}+1\right)}\right\}-\left\{1-2 \frac{\gamma_{1} \gamma_{2} \cdots \gamma_{r-1}}{\left(\gamma_{1}+1\right)\left(\gamma_{2}+1\right) \cdots\left(\gamma_{r-1}+1\right)}\right\}\right] \\
& \times\left[f_{Y_{2,2}}(y)-f_{Y_{1,1}}(y)\right] \\
& =\alpha\left[2\left\{\frac{\gamma_{1} \gamma_{2} \cdots \gamma_{r-1}}{\left(\gamma_{1}+1\right)\left(\gamma_{2}+1\right) \cdots\left(\gamma_{r-1}+1\right)}-\frac{\gamma_{1} \gamma_{2} \cdots \gamma_{r}}{\left(\gamma_{1}+1\right)\left(\gamma_{2}+1\right) \cdots\left(\gamma_{r}+1\right)}\right\}\right] \\
& \times\left[f_{Y_{2,2}}(y)-f_{Y_{1,1}}(y)\right] \\
& =2 \alpha\left[\frac{\gamma_{1} \gamma_{2} \cdots \gamma_{r-1}}{\left(\gamma_{1}+1\right)\left(\gamma_{2}+1\right) \cdots\left(\gamma_{r-1}+1\right)\left(\gamma_{r}+1\right)}\right]\left[f_{Y_{2,2}}(y)-f_{Y_{1,1}}(y)\right] .
\end{aligned}
$$

If $h(y)$ is a measurable function of $y$, then

$$
\begin{aligned}
E\left\{h\left(Y_{[r, n, m, k]}\right)\right\}-E\left\{h\left(Y_{[r-1, n, m, k]}\right)\right\}= & 2 \alpha\left[\frac{\gamma_{1} \gamma_{2} \cdots \gamma_{r-1}}{\left(\gamma_{1}+1\right)\left(\gamma_{2}+1\right) \cdots\left(\gamma_{r-1}+1\right)\left(\gamma_{r}+1\right)}\right] \\
& \times\left[E\left\{h\left(Y_{2,2}\right)\right\}-E\left\{h\left(Y_{1,1}\right)\right\}\right] .
\end{aligned}
$$

Hence, if we know $E\left\{h\left(Y_{[1, n, m, k]}\right)\right\}, E\left\{h\left(Y_{2,2}\right)\right\}$ and $E\left\{h\left(Y_{1,1}\right)\right\}$, then we can recursively calculate $E\left\{h\left(Y_{[2, n, m, k]}\right)\right\}, E\left\{h\left(Y_{[3, n, m, k]}\right)\right\}, \cdots, E\left\{h\left(Y_{[n, n, m, k]}\right)\right\}$.

Furthermore, we have from (4.5) with $i_{1}=1$,

$$
\begin{aligned}
g_{[r, n, m, k]}(y)-g_{[r, n-1, m, k]}(y)= & \alpha\left[C^{*}(r, n, m, k)-C^{*}(r, n-1, m, k)\right] \\
& \times\left[f_{Y_{2,2}}(y)-f_{Y_{1,1}}(y)\right] .
\end{aligned}
$$

Consider

$$
\begin{aligned}
C^{*}(r, n, m, k)-C^{*}(r, n-1, m, k) & =\left[1-2 \frac{\gamma_{1} \gamma_{2} \cdots \gamma_{r}}{\left(\gamma_{1}+1\right)\left(\gamma_{2}+1\right) \cdots\left(\gamma_{r}+1\right)}\right] \\
& -\left[1-2 \frac{\gamma_{1}^{*} \gamma_{2}^{*} \cdots \gamma_{r}^{*}}{\left(\gamma_{1}^{*}+1\right)\left(\gamma_{2}^{*}+1\right) \cdots\left(\gamma_{r}^{*}+1\right)}\right] \\
& =2\left[\frac{\gamma_{1}^{*} \gamma_{2}^{*} \cdots \gamma_{r}^{*}}{\left(\gamma_{1}^{*}+1\right)\left(\gamma_{2}^{*}+1\right) \cdots\left(\gamma_{r}^{*}+1\right)}-\frac{\gamma_{1} \gamma_{2} \cdots \gamma_{r}}{\left(\gamma_{1}+1\right)\left(\gamma_{2}+1\right) \cdots\left(\gamma_{r}+1\right)}\right] .
\end{aligned}
$$

Note that, $\gamma_{r}=k+(n-r)(m+1)$ and hence, $\gamma_{r}^{*}=k+(n-1-r)(m+1)=\gamma_{r+1}$. We now have

$$
\begin{aligned}
C^{*}(r, n, m, k)-C^{*}(r, n-1, m, k) & =2\left[\frac{\gamma_{2} \gamma_{3} \cdots \gamma_{r+1}}{\left(\gamma_{2}+1\right)\left(\gamma_{3}+1\right) \cdots\left(\gamma_{r+1}+1\right)}-\frac{\gamma_{1} \gamma_{2} \cdots \gamma_{r}}{\left(\gamma_{1}+1\right)\left(\gamma_{2}+1\right) \cdots\left(\gamma_{r}+1\right)}\right] \\
& =2\left[\frac{\gamma_{2} \gamma_{3} \cdots \gamma_{r}\left(\gamma_{r+1}-\gamma_{1}\right)}{\left(\gamma_{1}+1\right)\left(\gamma_{2}+1\right) \cdots\left(\gamma_{r}+1\right)\left(\gamma_{r+1}+1\right)}\right] \\
& =-2 r(m+1)\left[\frac{\gamma_{2} \gamma_{3} \cdots \gamma_{r}}{\left(\gamma_{1}+1\right)\left(\gamma_{2}+1\right) \cdots\left(\gamma_{r}+1\right)\left(\gamma_{r+1}+1\right)}\right] .
\end{aligned}
$$

Hence, from (4.19), we get

$$
\begin{aligned}
g_{[r, n, m, k]}(y)-g_{[r, n-1, m, k]}(y)= & \alpha\left[-2 r(m+1)\left\{\frac{\gamma_{2} \gamma_{3} \cdots \gamma_{r}}{\left(\gamma_{1}+1\right)\left(\gamma_{2}+1\right) \cdots\left(\gamma_{r}+1\right)\left(\gamma_{r+1}+1\right)}\right\}\right] \\
& \times\left[f_{Y_{2,2}}(y)-f_{Y_{1,1}}(y)\right] .
\end{aligned}
$$

5. Applications. The corresponding results for order statistics (with $m=0$ and $k=1$ ) and record values (with $m=-1$ and $k=-1$ ) for the 
bivariate FGM distributions (1.1) can easily be deduced as special cases of the results in Sections 2, 3 and 4.

\subsection{Order Statistics.}

Consider a random sample $\left(X_{i}, Y_{i}\right), i=1, \ldots, n$ from a bivariate distribution. If the pairs are ordered by their $X$ variates, then the $Y$ variate associated with the $r$-th order statistic $X_{r, n}$ of $X$ will be denoted by $Y_{[r, n]}, 1 \leq r \leq n$ and called the concomitant of the $r$-th order statistic. The pdf of $Y_{[r, n]}$, denoted by $g_{[r, n]}(y)$, is given by [David and Nagaraja (1983)]

$$
g_{[r, n]}(y)=\int_{-\infty}^{\infty} f_{Y \mid X}(y \mid x) f_{r, n}(x) d x
$$

where $f_{r, n}(x)$ is the pdf of $X_{r, n}$. Concomitants of order statistics have found a wide variety of applications in different fields. The most important use of concomitants arises in selection procedures when $k(1 \leq k \leq n)$ individuals are chosen on the basis of their $X$ values. Then the corresponding $Y$ values represent performance on an associated characteristic. For example, $X$ might be the score of a candidate on a screening test and $Y$ the score on a later test.

If we take $m=0$ and $k=1$, then

$$
C^{*}(r, n, m, k)=1-2 C(r, n, m, k)=1-2\left[\frac{\gamma_{1} \gamma_{2} \cdots \gamma_{r}}{\left(\gamma_{1}+1\right)\left(\gamma_{2}+1\right) \cdots\left(\gamma_{r}+1\right)}\right],
$$

where $\gamma_{r}=k+(n-r)(m+1)=n-r+1$, reduces to

$$
C^{*}(r, n, 0,1)=1-2 C(r, n, 0,1)=-\left(\frac{n-2 r+1}{n+1}\right) .
$$

Hence, from (2.34), the pdf of the concomitant of the $r$-th order statistic $Y_{[r, n]}$ is given by [Nair and Scaria (1999)]

$$
g_{[r, n]}(y)=f_{Y_{1,1}}(y)-\alpha\left(\frac{n-2 r+1}{n+1}\right)\left[f_{Y_{2,2}}(y)-f_{Y_{1,1}}(y)\right]
$$

which does not depend on $F_{X}(x)$. The $l$-th moment and mgf of $Y_{[r, n, m, k]}$ can be deduced from (3.1) and (3.3), respectively

$$
\mu_{[r, n]}^{(l)}=\left[1+\alpha\left(\frac{n-2 r+1}{n+1}\right)\right] \mu_{1,1}^{(l)}-\alpha\left(\frac{n-2 r+1}{n+1}\right) \mu_{2,2}^{(l)}
$$


and

$$
M_{[r, n]}(t)=\left[1+\alpha\left(\frac{n-2 r+1}{n+1}\right)\right] M_{1,1}(t)-\alpha\left(\frac{n-2 r+1}{n+1}\right) M_{2,2}(t),
$$

where $\mu_{[i, m]}^{(l)}=E\left\{Y_{[i, m]}^{l}\right\}$ is the $l$-th moment of $Y_{[i, m]}$ and $M_{[i, m]}(t)=E\left\{\exp \left(t Y_{[i, m]}\right)\right\}$ is the mgf of $Y_{[i, m]}$. Thus $\mu_{[r, n]}^{(l)}\left(M_{[r, n]}(t)\right)$ is known for all $r$ and $n$ if we know $\mu_{1,1}^{(l)}$ and $\mu_{2,2}^{(l)}\left(M_{1,1}(t)\right.$ and $\left.M_{2,2}(t)\right)$.

Furthermore, we can deduce several recurrence relations between pdf's, moments and mgf's of concomitants of order statistics from the results of Section 4. We have the following theorems.

THEOREM 5.1. Let $1 \leq i_{1} \leq i_{2} \leq n-r$ and $1 \leq j_{1} \leq j_{2} \leq r-1$. For $a$ bivariate random variable $(X, Y)$ having pdf (1.2), the following recurrence relations between pdf's of concomitants of order statistics (with $m=0$ and $k=1$ ) can be deduced using equations (3.9)-(3.12).

$$
\begin{aligned}
g_{[r, n]}(y)-g_{\left[r, n-i_{1}\right]}(y)= & \alpha\left[C^{*}(r, n, 0,1)-C^{*}\left(r, n-i_{1}, 0,1\right)\right] \\
& \times\left[f_{Y_{2,2}}(y)-f_{Y_{1,1}}(y)\right], \\
g_{[r, n]}(y)-g_{\left[r-j_{1}, n\right]}(y)= & \alpha\left[C^{*}(r, n, 0,1)-C^{*}\left(r-j_{1}, n, 0,1\right)\right] \\
& \times\left[f_{Y_{2,2}}(y)-f_{Y_{1,1}}(y)\right]
\end{aligned}
$$

and

$$
\begin{aligned}
g_{[r, n]}(y)-g_{\left[r-j_{1}, n-i_{1}\right]}(y)= & \alpha\left[C^{*}(r, n, 0,1)-C^{*}\left(r-j_{1}, n-i_{1}, 0,1\right)\right] \\
& \times\left[f_{Y_{2,2}}(y)-f_{Y_{1,1}}(y)\right] .
\end{aligned}
$$

In general it is true that

$$
\begin{aligned}
g_{\left[r-j_{1}, n-i_{1}\right]}(y) & -g_{\left[r-j_{2}, n-i_{2}\right]}(y)=\alpha\left[C^{*}\left(r-j_{1}, n-i_{1}, 0,1\right)\right. \\
& \left.-C^{*}\left(r-j_{2}, n-i_{2}, 0,1\right)\right]\left[f_{Y_{2,2}}(y)-f_{Y_{1,1}}(y)\right] .
\end{aligned}
$$

In particular with $i_{1}=1,(5.5)$ reduces to

$$
g_{[r, n]}(y)-g_{[r, n-1]}(y)=\alpha\left[\frac{2 r}{n(n+1)}\right]\left[f_{Y_{2,2}}(y)-f_{Y_{1,1}}(y)\right] .
$$


Moreover, from (5.9) by induction we get the following identity

$$
g_{[r, n]}(y)-g_{[r, r]}(y)=\alpha\left[\frac{2 r(n-r)}{(r+1)(n+1)}\right]\left[f_{Y_{2,2}}(y)-f_{Y_{1,1}}(y)\right] .
$$

Furthermore, with $j_{1}=1,(5.6)$ reduces to

$$
g_{[r, n]}(y)-g_{[r-1, n]}(y)=\left(\frac{2 \alpha}{n+1}\right)\left[f_{Y_{2,2}}(y)-f_{Y_{1,1}}(y)\right],
$$

which is independent of $r$.

If we change $n$ to $2 n+1$ and $r$ to $2 r$, then $\left(\frac{n-2 r+1}{n+1}\right)$ becomes $\left(\frac{2 n+1-4 r+1}{2 n+2}\right)=$ $\left(\frac{n-2 r+1}{n+1}\right)$ and hence from $(5.2)$

$$
g_{[r, n]}(y)=g_{[2 r, 2 n+1]}(y)
$$

which in turn implies

$$
\begin{gathered}
g_{[r, n]}(y)=g_{[2 r, 2 n+1]}(y)=g_{[4 r, 4 n+3]}(y)=g_{[8 r, 8 n+7]}(y)=\text { etc. } \\
=g_{\left[2^{k} r, 2^{k} n+2^{k}-1\right]}(y), \text { for } k=0,1,2, \ldots
\end{gathered}
$$

In general if $\lambda$ is a rational number such that $r \lambda$ and $(n+1) \lambda$ are integers, then

$$
g_{[r \lambda,(n+1) \lambda-1]}(y)=g_{[r, n]}(y) .
$$

This covers the previous result (5.12).

THEOREM 5.2. Under the conditions of Theorem 5.1, the relation between moments of concomitants of order statistics are given by

$$
\begin{aligned}
\mu_{[r, n]}^{(l)}-\mu_{\left[r, n-i_{1}\right]}^{(l)}= & \alpha\left[C^{*}(r, n, 0,1)-C^{*}\left(r, n-i_{1}, 0,1\right)\right] \\
& \times\left[\mu_{2,2}^{(l)}-\mu_{1,1}^{(l)}\right], \\
\mu_{[r, n]}^{(l)}-\mu_{\left[r-j_{1}, n\right]}^{(l)}= & \alpha\left[C^{*}(r, n, 0,1)-C^{*}\left(r-j_{1}, n, 0,1\right)\right] \\
& \times\left[\mu_{2,2}^{(l)}-\mu_{1,1}^{(l)}\right]
\end{aligned}
$$

and

$$
\begin{aligned}
\mu_{[r, n]}^{(l)}-\mu_{\left[r-j_{1}, n-i_{1}\right]}^{(l)}= & \alpha\left[C^{*}(r, n, 0,1)-C^{*}\left(r-j_{1}, n-i_{1}, 0,1\right)\right] \\
& \times\left[\mu_{2,2}^{(l)}-\mu_{1,1}^{(y)}\right]
\end{aligned}
$$


In general

$$
\begin{aligned}
\mu_{\left[r-j_{1}, n-i_{1}\right]}^{(l)} & -\mu_{\left[r-j_{2}, n-i_{2}\right]}^{(l)}=\alpha\left[C^{*}\left(r-j_{1}, n-i_{1}, 0,1\right)\right. \\
& \left.-C^{*}\left(r-j_{2}, n-i_{2}, 0,1\right)\right]\left[\mu_{2,2}^{(l)}-\mu_{1,1}^{(l)}\right] .
\end{aligned}
$$

In particular with $i_{1}=1,(5.14)$ reduces to

$$
\mu_{[r, n]}^{(l)}-\mu_{[r, n-1]}^{(l)}=\alpha\left[\frac{2 r}{n(n+1)}\right]\left[\mu_{2,2}^{(l)}-\mu_{1,1}^{(l)}\right] .
$$

Moreover, from (5.18) by induction we get the following identity

$$
\mu_{[r, n]}^{(l)}-\mu_{[r, r]}^{(l)}=\alpha\left[\frac{2 r(n-r)}{(r+1)(n+1)}\right]\left[\mu_{2,2}^{(l)}-\mu_{1,1}^{(l)}\right] .
$$

Furthermore, if $h(y)$ is a measurable function of $y$, then from (5.11) we get

$$
E\left\{h\left(Y_{[r, n]}\right)\right\}-E\left\{h\left(Y_{[r-1, n]}\right)\right\}=\left(\frac{2 \alpha}{n+1}\right)\left[E\left\{h\left(Y_{2,2}\right)\right\}-E\left\{h\left(Y_{1,1}\right)\right\}\right],
$$

which does not depend on $r$. Hence, if we know $E\left\{h\left(Y_{[1, n])}\right\}, E\left\{h\left(Y_{2,2}\right)\right\}\right.$ and $E\left\{h\left(Y_{1,1}\right)\right\}$, then we can recursively calculate $E\left\{h\left(Y_{[2, n]}\right)\right\}, E\left\{h\left(Y_{[3, n]}\right)\right\}, \ldots$, $E\left\{h\left(Y_{[n,: n]}\right)\right\}$.

THEOREM 5.3. Under conditions of Theorem 5.1, the relation between mgf of concomitants of order statistics are given by

$$
\begin{aligned}
M_{[r, n]}(t)-M_{\left[r, n-i_{1}\right]}(t)= & \alpha\left[C^{*}(r, n, 0,1)-C^{*}\left(r, n-i_{1}, 0,1\right)\right] \\
& \times\left[M_{2,2}(t)-M_{1,1}(t)\right], \\
M_{[r, n]}(t)-M_{\left[r-j_{1}, n\right]}(t)= & \alpha\left[C^{*}(r, n, 0,1)-C^{*}\left(r-j_{1}, n, 0,1\right)\right] \\
& \times\left[M_{2,2}(t)-M_{1,1}(t)\right]
\end{aligned}
$$

and

$$
\begin{aligned}
M_{[r, n]}(t)-M_{\left[r-j_{1}, n-i_{1}\right]}(t)= & \alpha\left[C^{*}(r, n, 0,1)-C^{*}\left(r-j_{1}, n-i_{1}, 0,1\right)\right] \\
& \times\left[M_{2,2}(t)-M_{1,1}(t)\right] .
\end{aligned}
$$

In general

$$
\begin{aligned}
M_{\left[r-j_{1}, n-i_{1}\right]}(t) & -M_{\left[r-j_{2}, n-i_{2}\right]}(t)=\alpha\left[C^{*}\left(r-j_{1}, n-i_{1}, 0,1\right)\right. \\
& \left.-C^{*}\left(r-j_{2}, n-i_{2}, 0,1\right)\right]\left[M_{2,2}(t)-M_{1,1}(t)\right] .
\end{aligned}
$$


Balasubramanian and Beg (1997) studied the concomitants of order statistics for Morgenstern type bivariate exponential distributions. Their results can be obtained from the results of this section with $F_{X}(x)=1-\exp (-x)$, $x>0$ and $F_{Y}(y)=1-\exp (-y), y>0$. Recently, Bairamov and Bekci (1999) have studied distribution and recurrence relations between moments of concomitants of order statistics in the bivariate FGM distributions with uniform marginals. Their results are special cases of the results of this section with $F_{X}(x)=x, 0<x<1$ and $F_{Y}(y)=y, 0<y<1$. The results on concomitants of order statistics corresponding to other marginals in FGM family can be obtained from the results of this section. More recently, Bairamov et al. (2001) have studied the concomitants of order statistics of the bivariate FGM distributions with uniform marginals by introducing additional parameters. For some subset of the parameters their results can be obtained from the results of this section.

\subsection{Record Values.}

Consider $\left(X_{1}, Y_{1}\right),\left(X_{2}, Y_{2}\right), \ldots$ a sequence of iid bivariate random variables. Variate $Y_{i}$ corresponding to $X_{i}=R_{r}$ ( $r$-th upper record) which will be represented by $Y_{\left[R_{r}\right]}$ is the concomitant of the $r$-th upper record. The pdf of $Y_{\left[R_{r}\right]}$ is given by

$$
g_{\left[R_{r}\right]}(y)=\int_{-\infty}^{\infty} f_{Y \mid X}(y \mid x) f_{R_{r}}(x) d x
$$

where $f_{R_{r}}(x)$ is the pdf of $R_{r}$.

If we take $m=-1$ and $k=1$, then

$$
C^{*}(r, n, m, k)=1-2 C(r, n, m, k)=1-2\left[\frac{\gamma_{1} \gamma_{2} \cdots \gamma_{r}}{\left(\gamma_{1}+1\right)\left(\gamma_{2}+1\right) \cdots\left(\gamma_{r}+1\right)}\right],
$$

where $\gamma_{r}=k+(n-r)(m+1)=1$, reduces to

$$
C^{*}(r, n,-1,1)=1-2 C(r, n,-1,1)=1-\frac{1}{2^{r-1}} .
$$

Hence, from (2.3), the pdf of the concomitant of the $r$-th record $Y_{\left[R_{r}\right]}$ is given by

$$
\left.g_{\left[R_{r}\right]}(y)=f_{Y_{1,1}}(y)+\alpha\left(1-\frac{1}{2^{r-1}}\right)\right]\left[f_{Y_{2,2}}(y)-f_{Y_{1,1}}(y)\right]
$$


which does not depend on $F_{X}(x)$. The $l$-th moment and mgf of $Y_{\left[R_{r}\right]}$ can be easily obtained from (3.1) and (3.3) respectively

$$
\mu_{\left[R_{r}\right]}^{(l)}=\left[1-\alpha\left(1-\frac{1}{2^{r-1}}\right)\right] \mu_{1,1}^{(l)}+\alpha\left(1-\frac{1}{2^{r-1}}\right) \mu_{2,2}^{(l)}
$$

and

$$
M_{\left[R_{r}\right]}(t)=\left[1-\alpha\left(1-\frac{1}{2^{r-1}}\right)\right] M_{1,1}(t)+\alpha\left(1-\frac{1}{2^{r-1}}\right) M_{2,2}(t),
$$

where $\mu_{\left[R_{r}\right]}^{(l)}=E\left\{Y_{\left[R_{r}\right]}^{l}\right\}$ is the $l$-th moment of $Y_{\left[R_{r}\right]}$ and $M_{\left[R_{r}\right]}(t)=E\left\{\exp \left(t Y_{\left[R_{r}\right]}\right)\right\}$ is the mgf of $Y_{\left[R_{r}\right]}$. Thus $\mu_{\left[R_{r}\right]}^{(l)}\left(M_{\left[R_{r}\right]}(t)\right)$ is known for all $r$ if we know $\mu_{1,1}^{(l)}$ and $\mu_{2,2}^{(l)}\left(M_{1,1}(t)\right.$ and $\left.M_{2,2}(t)\right)$.

Furthermore, we can deduce several recurrence relations between pdf's, moments and mgf's of concomitants of record values from the results of Section 4. We have the following theorems.

THEOREM 5.4. Let $1 \leq j_{1} \leq j_{2} \leq r-1$. For a bivariate random variable $(X, Y)$ having pdf (1.2) the following recurrence relations between pdf's of concomitants of record values (with $m=-1$ and $k=1$ ) can be deduced using equations (4.6) and (4.8).

$$
\begin{aligned}
g_{\left[R_{r}\right]}(y)-g_{\left[R_{r-j_{1}}\right]}(y)= & \alpha\left[C^{*}(r, n,-1,1)-C^{*}\left(r-j_{1}, n,-1,1\right)\right] \\
& \times\left[f_{Y_{2,2}}(y)-f_{Y_{1,1}}(y)\right]
\end{aligned}
$$

and in general

$$
\begin{aligned}
g_{\left[R_{r-j_{1}}\right]}(y) & -g_{\left[R_{r-j_{2}}\right]}(y)=\alpha\left[C^{*}\left(r-j_{1}, n,-1,1\right)\right. \\
& \left.-C^{*}\left(r-j_{2}, n,-1,1\right)\right]\left[f_{Y_{2,2}}(y)-f_{Y_{1,1}}(y)\right] .
\end{aligned}
$$

In particular with $j_{1}=1,(5.29)$ gives

$$
g_{\left[R_{r}\right]}(y)-g_{\left[R_{r-1}\right]}(y)=\alpha\left(\frac{1}{2^{r-1}}\right)\left[f_{Y_{2,2}}(y)-f_{Y_{1,1}}(y)\right] .
$$

THEOREM 5.5. Under the conditions of Theorem 5.4, the relation between moments of concomitants of record values are given by

$$
\begin{aligned}
\mu_{\left[R_{r}\right]}^{(l)}-\mu_{\left[R_{r-j_{1}}\right]}^{(l)}= & \alpha\left[C^{*}(r, n,-1,1)-C^{*}\left(r-j_{1}, n,-1,1\right)\right] \\
& \times\left[\mu_{2,2}^{(l)}-\mu_{1,1}^{(l)}\right]
\end{aligned}
$$


and in general

$$
\begin{aligned}
\mu_{\left[R_{r-j_{1}}\right]}^{(l)} & -\mu_{\left[R_{r-j_{2}}\right]}^{(l)}=\alpha\left[C^{*}\left(r-j_{1}, n,-1,1\right)\right. \\
& \left.-C^{*}\left(r-j_{2}, n,-1,1\right)\right]\left[\mu_{2,2}^{(l)}-\mu_{1,1}^{(l)}\right] .
\end{aligned}
$$

In particular with $j_{1}=1,(5.32)$ reduces to

$$
\mu_{\left[R_{r}\right]}^{(l)}-\mu_{\left[R_{r-1}\right]}^{(l)}=\left(\frac{\alpha}{2^{r-1}}\right)\left[\mu_{2,2}^{(l)}-\mu_{1,1}^{(l)}\right] .
$$

Moreover, if $h(y)$ is a measurable function of $y$, then from (5.31) we get

$$
\left.E\left\{h\left(Y_{\left[R_{r}\right]}\right)\right\}-E\left\{h\left(Y_{\left[R_{r-1}\right]}\right)\right\}=\left(\frac{\alpha}{2^{r-1}}\right)\right]\left[E\left\{h\left(Y_{2,2}\right)\right\}-E\left\{h\left(Y_{1,1}\right)\right\}\right] .
$$

Hence, if we know $E\left\{h\left(Y_{\left.\left[R_{1}\right]\right)}\right\}, E\left\{h\left(Y_{2,2}\right)\right\}\right.$ and $E\left\{h\left(Y_{1,1}\right)\right\}$, then $E\left\{h\left(Y_{\left[R_{r}\right]}\right)\right\}$ is known for all $r$.

Furthermore, from (5.34) by induction we get the following identity

$$
\mu_{\left[R_{r}\right]}^{(l)}=\mu_{\left[R_{1}\right]}^{(l)}+\alpha\left(1-\frac{1}{2^{r-1}}\right)\left[\mu_{2,2}^{(l)}-\mu_{1,1}^{(l)}\right] .
$$

THEOREM 5.6. Under the conditions of Theorem 5.4, the relation between mgf's of concomitants of record values are given by

$$
\begin{aligned}
M_{\left[R_{r}\right]}(t)-M_{\left[R_{r-j_{1}}\right]}(t)= & \alpha\left[C^{*}(r, n,-1,1)-C^{*}\left(r-j_{1}, n,-1,1\right)\right] \\
& \times\left[M_{2,2}(t)-M_{1,1}(t)\right]
\end{aligned}
$$

and in general

$$
\begin{aligned}
M_{\left[R_{r-j_{1}}\right]}(t) & -M_{\left[R_{r-j_{2}}\right]}(t)=\alpha\left[C^{*}\left(r-j_{1}, n,-1,1\right)\right. \\
& \left.-C^{*}\left(r-j_{2}, n,-1,1\right)\right]\left[M_{2,2}(t)-M_{1,1}(t)\right] .
\end{aligned}
$$

If we take $m=-1$ and $k$ is a positive integer greater than 1 , then we get the corresponding results of $k$ records.

5.3. Relation between distributions of the concomitants of recard values and order statistics.

The two expressions (5.2) and (5.26), for the concomitant of the $q$-th record and the concomitant of the $r$-th order statistic respectively are equivalent if

$$
\left[1-\frac{1}{2^{q-1}}\right]=-\left[\frac{n-2 r+1}{n+1}\right]
$$


or,

$$
r=(n+1)\left[1-\frac{1}{2^{q}}\right] .
$$

Since $r$ is an integer, $2^{q}$ should divide $(n+1)$. We can in fact take $n+1=2^{q}$, then $r=2^{q}-1$. Hence

$$
g_{\left[R_{m}\right]}(y)=g_{\left[2^{q}-1,2^{q}-1\right]}(y) .
$$

Thus, the distribution of concomitant of the $q$-th record of $X$ is the same as that of the concomitant of the maximum order statistic of $2^{q}-1$ observations of $X$ for the MGF distributions given in (1.1).

6. Joint distribution of two concomitants. In this section, we derive the joint distribution of concomitants of two generalized order statistics.

Let $Y_{[r, n, m, k]}$ and $Y_{[s, n, m, k]}$ be concomitants of the $r$-th and $s$-th generalized order statistics, respectively. Then the joint df and pdf of $Y_{[r, n, m, k]}$ and $Y_{[s, n, m, k]}$ are respectively given by

$$
G_{[r, s, n, m, k]}\left(y_{1}, y_{2}\right)=\int_{-\infty}^{\infty} \int_{-\infty}^{x_{2}} F_{Y \mid X}\left(y_{1} \mid x_{1}\right) F_{Y \mid X}\left(y_{2} \mid x_{2}\right) f_{r, s, n, m, k}\left(x_{1}, x_{2}\right) d x_{1} d x_{2}
$$

and

$$
g_{[r, s, n, m, k]}\left(y_{1}, y_{2}\right)=\int_{-\infty}^{\infty} \int_{-\infty}^{x_{2}} f_{Y \mid X}\left(y_{1} \mid x_{1}\right) f_{Y \mid X}\left(y_{2} \mid x_{2}\right) f_{r, s, n, m, k}\left(x_{1}, x_{2}\right) d x_{1} d x_{2},
$$

where $f_{r, s, n, m, k}\left(x_{1}, x_{2}\right)$ is the joint pdf of $(X(r, n, m, k), X(s, n, m, k)), 1 \leq$ $r<s \leq n$.

We first prove a lemma which will be useful in the sequel.

LEMMA 6.1. Let $p$ and $q$ be real numbers, then using notations of the previous sections, it is shown that

$$
\begin{aligned}
I_{p, q}= & \frac{c_{s-1}}{(r-1) !(s-r-1) !(m+1)^{s-2}} \int_{-\infty}^{\infty} \int_{-\infty}^{x_{2}}\left(\bar{F}_{X}\left(x_{1}\right)\right)^{p}\left(\bar{F}_{X}\left(x_{1}\right)\right)^{m}\left[1-\left(\bar{F}_{X}\left(x_{1}\right)\right)^{m+1}\right]^{r-1} \\
& \times\left[\left(\bar{F}_{X}\left(x_{1}\right)\right)^{m+1}-\left(\bar{F}_{X}\left(x_{2}\right)\right)^{m+1}\right]^{s-r-1} \\
& \times\left(\bar{F}_{X}\left(x_{2}\right)\right)^{\gamma_{s}-1}\left(\bar{F}_{X}\left(x_{2}\right)\right)^{q} f_{X}\left(x_{1}\right) f_{X}\left(x_{2}\right) d x_{1} d x_{2} \\
= & \frac{\gamma_{1} \gamma_{2} \cdots \gamma_{s}}{\left(\gamma_{1}+p+q\right)\left(\gamma_{2}+p+q\right) \cdots\left(\gamma_{r}+p+q\right)\left(\gamma_{r+1}+q\right) \cdots\left(\gamma_{s}+q\right)}
\end{aligned}
$$


PROOF. Making transformations $u=\left(\bar{F}_{X}\left(x_{1}\right)\right)^{m+1}$ and $v=\left(\bar{F}_{X}\left(x_{2}\right)\right)^{m+1}$ in (6.3), we get

$$
\begin{aligned}
I_{p, q}= & \frac{c_{s-1}}{(r-1) !(s-r-1) !(m+1)^{s}} \int_{0}^{1} \int_{0}^{u} u^{\frac{p}{m+1}}(1-u)^{r-1} \\
& \times(u-v)^{s-r-1} v^{\left(\gamma_{s+1}+q\right) /(m+1)} d v d u .
\end{aligned}
$$

Further, using transformation $v=u t$, the above equation gives

$$
\begin{aligned}
I_{p, q}= & \frac{c_{s-1}}{(r-1) !(s-r-1) !(m+1)^{s}} \int_{0}^{1} \int_{0}^{1} u^{\frac{p}{m+1}}(1-u)^{r-1} u^{s-r-1} \\
& \times(1-t)^{s-r-1}(u t)^{\frac{\gamma_{s+1}+q}{m+1}} u d t d u \\
= & \frac{c_{s-1}}{(r-1) !(s-r-1) !(m+1)^{s}} \int_{0}^{1}(1-u)^{r-1} u^{\frac{\gamma_{r}+p+q}{m+1}-1} d u \\
& \times \int_{0}^{1}(1-t)^{s-r-1} t^{\frac{\gamma_{s}+q}{m+1}-1} d t \\
= & \frac{c_{s-1}}{(r-1) !(s-r-1) !(m+1)^{s}} B\left(r, \frac{\gamma_{r}+p+q}{m+1}\right) B\left(s-r, \frac{\gamma_{s}+q}{m+1}\right) \\
= & \frac{c_{s-1}}{(r-1) !(s-r-1) !(m+1)^{s}} \frac{\Gamma(r) \Gamma\left(\frac{\gamma_{r}+p+q}{m+1}\right) \Gamma(s-r) \Gamma\left(\frac{\gamma_{s}+q}{m+1}\right)}{\Gamma\left(r+\frac{\gamma_{r}+p+q}{m+1}\right) \Gamma\left(s-r+\frac{\gamma_{s}+q}{m+1}\right)} \\
= & \frac{c_{s-1}}{(r-1) !(s-r-1) !(m+1)^{s}} \frac{(r-1) !(s-r-1) !}{\left(\frac{\gamma_{1}+p+q}{m+1}\right)\left(\frac{\gamma_{2}+p+q}{m+1}\right) \cdots\left(\frac{\gamma_{r}+p+q}{m+1}\right)} \\
& \times \frac{1}{\left(\frac{\gamma_{r+1}+q}{m+1}\right)\left(\frac{\gamma_{r+2}+q}{m+1}\right) \cdots\left(\frac{\gamma_{s}+q}{m+1}\right)} \\
= & \frac{\gamma_{1} \gamma_{2} \cdots \gamma_{s}}{\left(\gamma_{1}+p+q\right)\left(\gamma_{2}+p+q\right) \cdots\left(\gamma_{r}+p+q\right)\left(\gamma_{r+1}+q\right) \cdots\left(\gamma_{s}+q\right)} .
\end{aligned}
$$

Utilizing (1.3) and (1.7) in (6.1) and simplifying, we get

$$
\begin{aligned}
& G_{[r, s, n, m, k]}\left(y_{1}, y_{2}\right)=F_{Y}\left(y_{1}\right) F_{Y}\left(y_{2}\right)\left[1+\alpha \bar{F}_{Y}\left(y_{2}\right)\right. \\
& \times\left\{2 \int_{-\infty}^{\infty} \int_{-\infty}^{x_{2}} \bar{F}_{X}\left(x_{2}\right) f_{r, s, n, m, k}\left(x_{1}, x_{2}\right) d x_{1} d x_{2}-1\right\} \\
& +\alpha \bar{F}_{Y}\left(y_{1}\right)\left\{\int_{-\infty}^{\infty} \int_{-\infty}^{x_{2}}\left(2 \bar{F}_{X}\left(x_{1}\right)-1\right) f_{r, s, n, m, k}\left(x_{1}, x_{2}\right) d x_{1} d x_{2}\right\} \\
& +\alpha^{2} \bar{F}_{Y}\left(y_{1}\right) \bar{F}_{Y}\left(y_{2}\right)\left\{\int_{-\infty}^{\infty} \int_{-\infty}^{x_{2}}\left(2 \bar{F}_{X}\left(x_{1}\right)-1\right)\left(2 \bar{F}_{X}\left(x_{2}\right)-1\right)\right. \\
& \left.\left.\times f_{r, s, n, m, k}\left(x_{1}, x_{2}\right) d x_{1} d x_{2}\right\}\right]
\end{aligned}
$$




$$
\begin{aligned}
& =F_{Y}\left(y_{1}\right) F_{Y}\left(y_{2}\right)\left[1+\alpha \bar{F}_{Y}\left(y_{2}\right)\left\{2 \int_{-\infty}^{\infty} \int_{-\infty}^{x_{2}} \bar{F}_{X}\left(x_{2}\right)\right.\right. \\
& \left.\times f_{r, s, n, m, k}\left(x_{1}, x_{2}\right) d x_{1} d x_{2}-1\right\} \\
& +\alpha \bar{F}_{Y}\left(y_{1}\right)\left\{2 \int_{-\infty}^{\infty} \int_{-\infty}^{x_{2}} \bar{F}_{X}\left(x_{1}\right) f_{r, s, n, m, k}\left(x_{1}, x_{2}\right) d x_{1} d x_{2}-1\right\} \\
& +\alpha^{2} \bar{F}_{Y}\left(y_{1}\right) \bar{F}_{Y}\left(y_{2}\right)\left\{\int_{-\infty}^{\infty} \int_{-\infty}^{x_{2}}\left[4 \bar{F}_{X}\left(x_{1}\right) \bar{F}_{X}\left(x_{2}\right)-2 \bar{F}_{X}\left(x_{1}\right)-2 \bar{F}_{X}\left(x_{2}\right)+1\right]\right. \\
& \left.\left.\times f_{r, s, n, m, k}\left(x_{1}, x_{2}\right) d x_{1} d x_{2}\right\}\right] \\
& =F_{Y}\left(y_{1}\right) F_{Y}\left(y_{2}\right)\left[1+\alpha \bar{F}_{Y}\left(y_{2}\right)\left[2 I_{0,1}-1\right]+\alpha \bar{F}_{Y}\left(y_{1}\right)\left[2 I_{1,0}-1\right]\right. \\
& \left.+\alpha^{2} \bar{F}_{Y}\left(y_{1}\right) \bar{F}_{Y}\left(y_{2}\right)\left[4 I_{1,1}-2 I_{1,0}-2 I_{0,1}+1\right]\right]
\end{aligned}
$$

Substituting values of $I_{0,1}, I_{1,0}$ and $I_{1,1}$, respectively, from Lemma 6.1 in (6.4), we obtain the joint df of $Y_{[r, n, m, k]}$ and $Y_{[s, n, m, k]}$,

$$
\begin{aligned}
G_{[r, s, n, m, k]}\left(y_{1}, y_{2}\right)= & F_{Y}\left(y_{1}\right) F_{Y}\left(y_{2}\right)\left[1+\alpha \bar{F}_{Y}\left(y_{2}\right)\left[2\left\{\frac{\gamma_{1} \gamma_{2} \cdots \gamma_{s}}{\left(\gamma_{1}+1\right)\left(\gamma_{2}+1\right) \cdots\left(\gamma_{s}+1\right)}\right\}-1\right]\right. \\
& +\alpha \bar{F}_{Y}\left(y_{1}\right)\left[2\left\{\frac{\gamma_{1} \gamma_{2} \cdots \gamma_{r}}{\left(\gamma_{1}+1\right)\left(\gamma_{2}+1\right) \cdots\left(\gamma_{r}+1\right)}\right\}-1\right] \\
& +\alpha^{2} \bar{F}_{Y}\left(y_{1}\right) \bar{F}_{Y}\left(y_{2}\right)\left[4\left\{\frac{\gamma_{1} \gamma_{2} \cdots \gamma_{r} \gamma_{r+1} \cdots \gamma_{s}}{\left(\gamma_{1}+2\right)\left(\gamma_{2}+2\right) \cdots\left(\gamma_{r}+2\right)\left(\gamma_{r+1}+1\right) \cdots\left(\gamma_{s}+1\right)}\right\}\right. \\
& \left.\left.-2\left\{\frac{\gamma_{1} \gamma_{2} \cdots \gamma_{r}}{\left(\gamma_{1}+1\right)\left(\gamma_{2}+1\right) \cdots\left(\gamma_{r}+1\right)}\right\}-2\left\{\frac{\gamma_{1} \gamma_{2} \cdots \gamma_{s}}{\left(\gamma_{1}+1\right)\left(\gamma_{2}+1\right) \cdots\left(\gamma_{s}+1\right)}\right\}+1\right]\right] .
\end{aligned}
$$

The pdf corresponding to $(6.5)$ is

$$
\begin{aligned}
g_{[r, s, n, m, k]}\left(y_{1}, y_{2}\right)= & f_{Y}\left(y_{1}\right) f_{Y}\left(y_{2}\right)\left[1+\alpha\left(2 \bar{F}_{Y}\left(y_{2}\right)-1\right)\left[2\left\{\frac{\gamma_{1} \gamma_{2} \cdots \gamma_{s}}{\left(\gamma_{1}+1\right)\left(\gamma_{2}+1\right) \cdots\left(\gamma_{s}+1\right)}\right\}-1\right]\right. \\
& +\alpha\left(2 \bar{F}\left(y_{1}\right)-1\right)\left[2\left\{\frac{\gamma_{1} \gamma_{2} \cdots \gamma_{r}}{\left(\gamma_{1}+1\right)\left(\gamma_{2}+1\right) \cdots\left(\gamma_{r}+1\right)}\right\}-1\right] \\
& +\alpha^{2}\left(2 \bar{F}\left(y_{1}\right)-1\right)\left(2 \bar{F}\left(y_{2}\right)-1\right) \\
& \times\left[4\left\{\frac{\gamma_{1} \gamma_{2} \cdots \gamma_{r} \gamma_{r+1} \cdots \gamma_{s}}{\left(\gamma_{1}+2\right)\left(\gamma_{2}+2\right) \cdots\left(\gamma_{r}+2\right)\left(\gamma_{r+1}+1\right) \cdots\left(\gamma_{s}+1\right)}\right\}\right. \\
& \left.\left.-2\left\{\frac{\gamma_{1} \gamma_{2} \cdots \gamma_{r}}{\left(\gamma_{1}+1\right)\left(\gamma_{2}+1\right) \cdots\left(\gamma_{r}+1\right)}\right\}-2\left\{\frac{\gamma_{1} \gamma_{2} \cdots \gamma_{s}}{\left(\gamma_{1}+1\right)\left(\gamma_{2}+1\right) \cdots\left(\gamma_{s}+1\right)}\right\}+1\right]\right] .
\end{aligned}
$$

\subsection{Applications.}

The joint df and pdf of concomitants of the $r$-th and $s$-th order statistics, $Y_{[r, n]}$ and $Y_{[s, n]}$, can easily be deduced from (6.5) and (6.6) respectively, with $m=0, k=1$ and $\gamma_{j}=n-j+1$.

First we evaluate $I_{0,1}, I_{1,0}$ and $I_{1,1}$ for order statistics.

$$
I_{0,1}=\frac{\gamma_{1} \gamma_{2} \cdots \gamma_{r} \gamma_{r+1} \cdots \gamma_{s}}{\left(\gamma_{1}+1\right)\left(\gamma_{2}+1\right) \cdots\left(\gamma_{r}+1\right)\left(\gamma_{r+1}+1\right) \cdots\left(\gamma_{s}+1\right)}=\frac{(n-s+1)}{(n+1)}
$$




$$
I_{1,0}=\frac{\gamma_{1} \gamma_{2} \cdots \gamma_{r}}{\left(\gamma_{1}+1\right)\left(\gamma_{2}+1\right) \cdots\left(\gamma_{r}+1\right)}=\frac{(n-r+1)}{(n+1)}
$$

and

$$
\begin{aligned}
I_{1,1} & =\frac{\gamma_{1} \gamma_{2} \cdots \gamma_{r-1} \gamma_{r} \gamma_{r+1} \cdots \gamma_{s-1} \gamma_{s}}{\left(\gamma_{1}+2\right)\left(\gamma_{2}+2\right) \cdots\left(\gamma_{r-1}+2\right)\left(\gamma_{r}+2\right)\left(\gamma_{r+1}+1\right) \cdots\left(\gamma_{s}+1\right)} \\
& =\frac{(n-s+1)(n-r+2)}{(n+1)(n+2)} .
\end{aligned}
$$

We now have

$$
\begin{aligned}
& 2 I_{0,1}-1=\frac{(n-2 s+1)}{(n+1)}, \\
& 2 I_{1,0}-1=\frac{(n-2 r+1)}{(n+1)}
\end{aligned}
$$

and

$$
\begin{aligned}
4 I_{1,1}-2 I_{0,1}-2 I_{1,0}+1 & =4\left[\frac{(n-r+2)(n-s+1)}{(n+1)(n+2)}\right]-2\left[\frac{(n-s+1)}{(n+1)}\right] \\
& -2\left[\frac{(n-r+1)}{(n+1)}\right]+1 \\
& =\frac{2(n-s+1)}{(n+1)}\left[\frac{2(n-r+2)}{(n+2)}-1\right] \\
& -\frac{2(n-r+1)}{(n+1)}+1 \\
& =\frac{(n-2 s+1)}{(n+1)(n+2)}[(n+2)-2 r]+\frac{2 r}{(n+1)(n+2)} \\
& =\frac{(n-2 s+1)}{(n+1)}-\frac{2 r(n-2 s)}{(n+1)(n+2)} .
\end{aligned}
$$

Substituting the above values in (6.5) and (6.6), we get the joint df and pdf of $Y_{[r, n]}$ and $Y_{[s, n]}$, respectively [Nair and Scaria (1999)]

$$
\begin{aligned}
G_{[r, s, n]}\left(y_{1}, y_{2}\right)= & F_{Y}\left(y_{1}\right) F_{Y}\left(y_{2}\right)\left[1+\alpha \bar{F}_{Y}\left(y_{1}\right) \frac{(n-2 r+1)}{(n+1)}+\alpha \bar{F}_{Y}\left(y_{2}\right)\right. \\
& \left.\times \frac{(n-2 s+1)}{(n+1)}+\alpha^{2} \bar{F}_{Y}\left(y_{1}\right) \bar{F}_{Y}\left(y_{2}\right)\left\{\frac{(n-2 s+1)}{(n+1)}-\frac{2 r(n-2 s)}{(n+1)(n+2)}\right\}\right]
\end{aligned}
$$

and

$$
\begin{aligned}
& g_{[r, s, n]}\left(y_{1}, y_{2}\right)=f_{Y}\left(y_{1}\right) f_{Y}\left(y_{2}\right)\left[1+\alpha\left(2 \bar{F}_{Y}\left(y_{1}\right)-1\right) \frac{(n-2 r+1)}{(n+1)}\right. \\
& +\alpha\left(2 \bar{F}_{Y}\left(y_{2}\right)-1\right) \frac{(n-2 s+1)}{(n+1)}+\alpha^{2}\left(2 \bar{F}_{Y}\left(y_{1}\right)-1\right)\left(2 \bar{F}_{Y}\left(y_{2}\right)-1\right) \\
& \left.\times\left\{\frac{(n-2 s+1)}{(n+1)}-\frac{2 r(n-2 s)}{(n+1)(n+2)}\right\}\right]
\end{aligned}
$$


Also, the joint df and pdf of the concomitants of the $r$-th and $s$-th record values, $Y_{\left[R_{r}\right]}$ and $Y_{\left[R_{s}\right]}$, can be deduced from (6.5) and (6.6), respectively, with $m=-1, k=1$ and $\gamma_{j}=1$.

First we evaluate $I_{0,1}, I_{1,0}$ and $I_{1,1}$ for record values.

$$
\begin{aligned}
I_{0,1} & =\frac{\gamma_{1} \gamma_{2} \cdots \gamma_{r} \gamma_{r+1} \cdots \gamma_{s}}{\left(\gamma_{1}+1\right)\left(\gamma_{2}+1\right) \cdots\left(\gamma_{r}+1\right)\left(\gamma_{r+1}+1\right) \cdots\left(\gamma_{s}+1\right)}=\frac{1}{2^{s}}, \\
I_{1,0} & =\frac{\gamma_{1} \gamma_{2} \cdots \gamma_{r}}{\left(\gamma_{1}+1\right)\left(\gamma_{2}+1\right) \cdots\left(\gamma_{r}+1\right)}=\frac{1}{2^{r}}
\end{aligned}
$$

and

$$
\begin{aligned}
I_{1,1} & =\frac{\gamma_{1} \gamma_{2} \cdots \gamma_{r-1} \gamma_{r} \gamma_{r+1} \cdots \gamma_{s-1} \gamma_{s}}{\left(\gamma_{1}+2\right)\left(\gamma_{2}+2\right) \cdots\left(\gamma_{r-1}+2\right)\left(\gamma_{r}+2\right)\left(\gamma_{r+1}+1\right) \cdots\left(\gamma_{s}+1\right)} \\
& =\frac{1}{3^{r} 2^{s-r}} .
\end{aligned}
$$

We now have

$$
\begin{aligned}
& 2 I_{0,1}-1=\frac{1}{2^{s-1}}-1, \\
& 2 I_{1,0}-1=\frac{1}{2^{r-1}}-1
\end{aligned}
$$

and

$$
4 I_{1,1}-2 I_{0,1}-2 I_{1,0}+1=\frac{1}{3^{r} 2^{s-r-2}}-\frac{1}{2^{s-1}}-\frac{1}{2^{r-1}}+1 .
$$

Substituting the above values in (6.5) and (6.6), we get the joint df and pdf of $Y_{\left[R_{r}\right]}$ and $Y_{\left[R_{s}\right]}$, respectively

$$
\begin{aligned}
& G_{\left[R_{r}, R_{s}\right]}\left(y_{1}, y_{2}\right)=F_{Y}\left(y_{1}\right) F_{Y}\left(y_{2}\right)\left[1+\alpha \bar{F}_{Y}\left(y_{1}\right)\left\{\frac{1}{2^{r-1}}-1\right\}\right. \\
& \left.+\alpha \bar{F}_{Y}\left(y_{2}\right)\left\{\frac{1}{2^{s-1}}-1\right\}+\alpha^{2} \bar{F}_{Y}\left(y_{1}\right) \bar{F}_{Y}\left(y_{2}\right)\left\{\frac{1}{3^{r} 2^{s-r-2}}-\frac{1}{2^{s-1}}-\frac{1}{2^{r-1}}+1\right\}\right]
\end{aligned}
$$

and

$$
\begin{aligned}
& g_{\left[R_{r}, R_{s}\right]}\left(y_{1}, y_{2}\right)=f_{Y}\left(y_{1}\right) f_{Y}\left(y_{2}\right)\left[1+\alpha\left(2 \bar{F}_{Y}\left(y_{1}\right)-1\right)\left\{\frac{1}{2^{r-1}}-1\right\}\right. \\
& +\alpha\left(2 \bar{F}_{Y}\left(y_{2}\right)-1\right)\left\{\frac{1}{2^{s-1}}-1\right\}+\alpha^{2}\left(2 \bar{F}_{Y}\left(y_{1}\right)-1\right) \\
& \left.\times\left(2 \bar{F}_{Y}\left(y_{2}\right)-1\right)\left\{\frac{1}{3^{r} 2^{s-r-2}}-\frac{1}{2^{s-1}}-\frac{1}{2^{r-1}}+1\right\}\right] .
\end{aligned}
$$

7. Product moments of $Y_{[r, n, m, k]}$ and $Y_{[r, n, m, k]}$. With the joint density $g_{[r, s, n, m, k]}\left(y_{1}, y_{2}\right)$ of $Y_{[r, n, m, k]}$ and $Y_{[s, n, m, k]}$, the product moments 
$E\left\{Y_{[r, n, m, k]}^{l_{1}} Y_{[s, n, m, k]}^{l_{2}}\right\}$, denoted by $\mu_{[r, s, n, m, k}^{\left(l_{1}, l_{2}\right)}, l_{1}, l_{2}>0$, are given by

$$
\mu_{[r, s, n, m, k]}^{\left(l_{1}, l_{2}\right)}=\int_{-\infty}^{\infty} \int_{-\infty}^{\infty} g_{[r, s, n, m, k]}\left(y_{1}, y_{2}\right) d y_{1} d y_{2}
$$

Utilizing (6.6) in (7.1) and simplifying, we get

$$
\begin{aligned}
\mu_{[r, s, n, m, k]}^{\left(l_{1}, l_{2}\right)} & =\mu_{1,1}^{l_{1}} \mu_{1,1}^{l_{2}}+\alpha\left[\mu_{1,1}^{l_{1}} \mu_{1,1}^{l_{2}}-\mu_{1,1}^{l_{1}} \mu_{2,2}^{l_{2}}\right]\left[2 I_{0,1}-1\right] \\
& +\alpha\left[\mu_{1,1}^{l_{1}} \mu_{1,1}^{l_{2}}-\mu_{2,2}^{l_{1}} \mu_{1,1}^{l_{2}}\right]\left[2 I_{1,0}-1\right] \\
& +\alpha^{2}\left[\mu_{1,1}^{l_{1}}-\mu_{2,2}^{l_{1}}\right]\left[\mu_{1,1}^{l_{2}}-\mu_{2,2}^{l_{2}}\right]\left[4 I_{1,1}-2 I_{0,1}-2 I_{1,0}+1\right] .
\end{aligned}
$$

Furthermore, using (3.1) and (7.2), the covariance of $Y_{[r, n, m, k]}$ and $Y_{[r, n, m, k]}$

$$
\operatorname{Cov}\left(Y_{[r, n, m, k]}, Y_{[r, n, m, k]}\right)=\mu_{[r, s, n, m, k]}-\mu_{[r, n, m, k]} \mu_{[s, n, m, k]}, r \neq s
$$

The joint mgf of $Y_{[r, n, m, k]}$ and $Y_{[r, n, m, k]}$, is given by

$$
\begin{aligned}
M_{[r, s, n, m, k]}\left(t_{1}, t_{2}\right) & =E\left\{\exp \left(t_{1} Y_{[r, n, m, k]}+t_{2} Y_{[s, n, m, k]}\right)\right\} \\
& =\int_{-\infty}^{\infty} \int_{-\infty}^{\infty} \exp \left\{t_{1} y_{1}+t_{2} y_{2}\right\} g_{[r, s, n, m, k]}\left(y_{1}, y_{2}\right) d y_{1} d y_{2} .
\end{aligned}
$$

Utilizing (6.6) in (7.4) and simplifying, we get

$$
\begin{aligned}
& M_{[r, s, n, m, k]}\left(t_{1}, t_{2}\right)=M_{Y_{1,1}}\left(t_{1}\right) M_{Y_{1,1}}\left(t_{2}\right) \\
& +\alpha\left[M_{Y_{1,1}}\left(t_{1}\right) M_{Y_{1,1}}\left(t_{2}\right)-M_{Y_{1,1}}\left(t_{1}\right) M_{Y_{2,2}}\left(t_{2}\right)\right]\left[2 I_{0,1}-1\right] \\
& +\alpha\left[M_{Y_{1,1}}\left(t_{1}\right) M_{Y_{1,1}}\left(t_{2}\right)-M_{Y_{2,2}}\left(t_{1}\right) M_{Y_{1,1}}\left(t_{2}\right)\right]\left[2 I_{1,0}-1\right] \\
& +\alpha^{2}\left[M_{Y_{1,1}}\left(t_{1}\right)-M_{Y_{2,2}}\left(t_{1}\right)\right]\left[M_{Y_{1,1}}\left(t_{2}\right)-M_{Y_{2,2}}\left(t_{2}\right)\right]\left[4 I_{1,1}-2 I_{0,1}-2 I_{1,0}+1\right] .
\end{aligned}
$$

Differentiating (7.5) with respect to $t_{1}$ and $t_{2}, l_{1}$ times and $l_{2}$ times, respectively, and putting $t_{1}=t_{2}=0$, we get (7.2).

From (7.2) and (7.5) one can deduce product moments and joint mgf's for order statistics (with $m=0$ and $k=1$ ) and record values (with $m=-1$ and $k=1$ ), respectively.

REMARK. We can obtain results for concomitants of generalized order statistics, order statistics and record values corresponding to different bivariate distributions of FGM family by specifying the respective marginal distributions from the general results of this paper.

Acknowledgments. The authors thank Professor K. Balasubramanian and Professor Y.P. Chaubey for valuable discussions and suggestions. 


\section{REFERENCES}

AHSANUlLAH, M. (2004). Record Values-Theory and Applications. Univ. Press of America, Lanham, Maryland.

BAIRANOV, I.G. and BEKCI, M. (1999). Concomitant of order statistics in FGM type bivariate uniform distributions. Istatistik, J. Turkish Statistical Association 2 135-144.

BAIRANOV, I.G., KOTZ, S. and BEKCI, M. (2001). New generalized Farlie-Gumbel-Morgenstern distributions and concomitants of order statistics. J. Appl. Statist. 28 521-536.

BALASUBRAMANIAN, K. and Beg, M.I. (1997). Concomitant of order statistics in Morgenstern type bivariate exponential distributions. $J$. Appl. Statistical Science 5 233-245.

BHTTACHARYA, P.K. (1984). Induced order statistics. In: P.R. Krishnaiah and P.K. Sen (eds.): Handbook of Statistics. 4 383-403.

DAVID, H.A. and NAGARAJA, H.N. (1998). Concomitant of order statistics. In: N. Balakrishnan and C.R. Rao (eds.): Handbook of Statistics. 16 487-513.

DAVID, H.A. and NAGARAJA, H.N. (2003). Order Statistics. 3rd ed. Wiley, New York.

FARLIE, D.J.G. (1960). The performance of some correlation coefficients for a general bivariate distribution. Biometrika 47 307-323.

GUMBEL, E.J. (1960). Bivariate exponential distributions. J. Amer. Statist. Assoc. 55 698-707.

JOHNSON, N.L. and KOTZ, S. (1975). On some generalized Farlie-GumbelMorgenstern distributions. Commun. statist. Theor. Meth. 4 415-427.

KAMPS, U. (1956). A Concept of Generalized Order Statistics. Teubner, Stuttgart.

MORGENSTERN, D. (1956). Einfache Beispiele Zweidimensionaler Verteilungen. Mitteilungsblatt fur Mathematische Statistik 8 234-235.

SCARIA, J. and NAIR, N.U. (1999). On concomitants of order statistics from Morgenstern family. Biomet. J. 41 483-489.

DEPARTMENT OF MATHEMATICS
AND STATISTICS
UNIVERSITY OF HYDERABAD
HYDERABAD 500046
INDIA
E-MAIL: m_i_beg@yahoo.com

DEPARTMENT OF MATHEMATICS AND STATISTICS

UNIVERSITY OF HYDERABAD

HYDERABAD 500046

E-MAIL: m_i_beg@yahoo.com

\author{
DEPARTMENT OF MANAGEMENT \\ SCIENCES \\ RIDER UNIVERSITY \\ LAWRENCEVILLE, NJ 08648-3099 \\ USA \\ E-MAIL: ahsan@rider.edu
}




\section{List of Recent Technical Reports}

46. Eduardo Rodriguez, Scoring Methods for Risk Classification, April 2000

47. R. Rodríguez-Ramos, L. Cortés-Carrasquero, J. Bravo-Castillero and R. Guinovart-Díaz, Homogeneization in Elasto-plastic Composites, June 2000

48. A. A. Alvarez-Mesquida, R. Rodríguez-Ramos, F. Comas, R. Esquivel and G. Monsivais, Scattering of Shear Horizontal Elastic Waves in a Heterogeneous Piezoelectric Medium, June 2000

49. M. L. Filshtinsky, R. Rodríguez-Ramos and O. Sanchez-Casals, Fracture Mechanic in Piezoceramic Composite Plate, June 2000

50. F. Lebon, R. Rodriguez-Ramos and A. Mesejo, Homogenization and Wavelet-Galerkin Method for a Nonlinear One-dimensional Problem, June 2000

51. L. Yang, The Impact of Mortality Improvement on Social Security, August 2000

52. Rodrigo Arias López and José Garrido, Bounds and Other Properties of the Inverse, Moments of a Positive Binomial Variate, September 2000

53. B. N. Dimitrov, Z. Khalil, M. E. Ghitany and V. V. Rykov, Likelihood Ratio Test for Almost Lack of Memory Distributions, November 2001

54. Yogendra P. Chaubey and Anthony Crisalli, The Generalized Smoothing Estimator, April 2002

55. Yogendra P. Chaubey and Pranab K. Sen, Smooth Isotonic Estimation of Density, Hazard and MRL Functions, April 2002

56. Pablo Olivares, Maximum Likelihood Estimators for a Branching-Diffusion Process, August 2002

57. Shuanming Li and José Garrido, On Ruin for the Erlang(n) Risk Process, June 2003 
58. G. Jogesh Babu and Yogendra P. Chaubey, Smooth Estimation of a Distribution and Density function on a Hypercube Using Bernstein Polynomials for Dependent Random Vectors , August 2003

59. Shuanming Li and José Garrido, On the Time Value of Ruin for a Sparre Anderson Risk Process Perturbed by Diffusion, November 2003

60. Yogendra P. Chaubey, Cynthia M. DeSouza and Fassil Nebebe, Bayesian Inference for Small Area Estimation under the Inverse Gaussian Model via Cibbs Sampling, December 2003

61. Alexander Melnikov and Victoria Skornyakova, Pricing of EquityLinked Life Insurance Contracts with Flexible Guarantees, May 2004

62. Yi Lu and José Garrido, Regime-Switching Periodic Models for Claim Counts, June 2004.

63. I. Urrutia-Romaní, R. Rodríguez-Ramos, J. Bravo-Castillero and R. Guinovart-Díaz, Asymptotic Homogenization Method Applied to Linear Viscoelastic Composites. Examples, August 2004.

64. Yi Lu and José Garrido, Double Periodic Non-Homogeneous Poisson Models for Hurricanes Data, September 2004.

65. M.I. Beg and M. Ahsanullah, On Characterizing Distributions by Conditional Expectations of Functions of Generalized Order Statistics, September, 2004.

66. M.I. Beg and M. Ahsanullah, Concomitants of Generalized Order Statistics from Farlie-Gumbel-Morgenstern Distributions, September, 2004.

Copies of technical reports can be requested from:

Prof. Xiaowen Zhou

Department of Mathematics and Statistics

Concordia University

7141, Sherbrooke Street West

Montréal (QC) H4B 1R6 CANADA 